\title{
Genus distributions of graphs under edge-amalgamations
}

\author{
Mehvish I. Poshni , Imran F. Khan , Jonathan L. Gross \\ Columbia University, Department of Computer Science NY 10027, New York, USA
}

Received 2 July 2009, accepted 17 February 2010, published online 19 March 2010

\begin{abstract}
We present a general method for calculating the genus distributions of those infinite families of graphs that are obtained by iteratively amalgamating copies of some base graphs along their root-edges. We presume that the partitioned genus distributions of these base graphs are known and that their root-edges have 2-valent endpoints. We analyze and adapt the use of recombinant strands, partials, and productions for deriving simultaneous recurrences for genus distributions.
\end{abstract}

Keywords: Graph, genus distribution, edge-amalgamation.

Math. Subj. Class.: 05C10

\section{Introduction}

In this paper, we illustrate a general method that enables us to deal with recursively defined infinite families of graphs, the calculation of whose genus distributions has not been hitherto possible without the new methods in this paper. In particular, we demonstrate how to calculate the genus distribution of an arbitrary chain of copies of one or more graphs, that results from the iterative amalgamation along their root-edges. This may be done for edge-linked chains constructed by using copies of different types of graphs or by using multiple copies of the same graph. We can produce genus distributions for various infinite families of 3-regular graphs in this manner, apart from many other infinite classes. Another contribution of this paper is an easily understood method in $\S 6$ for constructing pairs of non-homeomorphic graphs with the same genus distribution. Moreover, the results of this paper are used by [9] to construct a quadratic-time algorithm for calculating the genus distribution of any 3-regular outerplanar graph.

Prior research on counting imbeddings on various orientable and non-orientable surfaces includes [3], [4], [5], [10], [11], [13], [16], [17], [18], [19], [21], [23], [24], [25],

E-mail addresses: poshni@cs.columbia.edu (Mehvish I. Poshni), imran@cs.columbia.edu (Imran F. Khan), gross@cs.columbia.edu (Jonathan L. Gross) 
[26], [27], [28], [29], [30], and [31]. Prior work on counting graph imbeddings in a minimum-genus surface includes [2], [7], [6], and [15]. The second installment of this paper [22] demonstrates how to calculate the genus distribution of a graph that results from self-amalgamation along its root-edges.

In this paper we assume a basic background and familiarity with topological graph theory (see [14] or [32]). We denote an orientable surface of genus $i$ by $S_{i}$ and the number of imbeddings of a graph $G$ on the surface $S_{i}$ by $g_{i}(G)$. Unless indicated otherwise, an imbedding will be considered 2-cellular and orientable, and a graph will be considered connected. We use the abbreviation $\boldsymbol{f b}$-walk for face-boundary walk. We allow a graph to contain multiple adjacencies and self-loops. We refer the reader to [14] or [1] for a more detailed guide to the terminology assumed here.

We designate two edges of a graph as the root-edges or roots of a double-edge-rooted graph. We presently require a root-edge to have 2 -valent endpoints. The edge-amalgamation of a pair of double-rooted graphs $(G, e, d)$ and $(H, g, f)$ is the graph obtained by merging the roots $d$ and $g$. We denote this operation by an asterisk:

$$
(G, e, d) *(H, g, f)=(W, e, f)
$$

where $W$ is the merged graph and $e$ and $f$ are its roots. There are two different ways of amalgamating edges $d$ and $g$, depending on how the endpoints of $d$ are paired up with the endpoints of $g$. This information is not captured in our notation, and it is obvious from context what is intended for a particular scenario. Insofar as the genus distributions are concerned, we will establish in this paper that graphs resulting from either way of edgeamalgamation have identical genus distributions.

The definition of edge-amalgamation for graphs carries over naturally to the edgeamalgamation of graph imbeddings. The imbeddings of the graph $W=G * H$ are obtained by combining the rotation systems for the graphs $G$ and $H$ in all possible ways. Thus, each imbedding $\iota_{W}$ of the graph $W$ induces unique imbeddings $\iota_{G}$ and $\iota_{H}$ of the graphs $G$ and $H$, respectively, such that the rotation system corresponding to $\iota_{W}$ is consistent with the rotation systems corresponding to $\iota_{G}$ and $\iota_{H}$.

Another useful concept is that of a strand, which we define to be an open subwalk of an fb-walk that runs between any two occurrences of the endpoints of a root-edge $e$, such that there are no occurrences of the edge $e$ or the endpoints of $e$ in its interior.

We analyze the effects of amalgamating two graph imbeddings by using rules called productions, which we describe later.

\section{Partitioned genus distributions}

In order to explain what a production is, we first describe ways to categorize an imbedding of a double-rooted graph. We are primarily interested here in the fb-walks incident on the root-edges, as the crux of our work focuses on how these fb-walks change in response to the amalgamation operation on the graphs. Each root-edge has two 2-valent endpoints, so each root has either two distinct face-boundaries incident on it, or the same fb-walk is incident on both sides of it. Accordingly, we use the mnemonic $d$ for double and $s$ for single in defining the double-root partials in Table 1. Note that the subscript $i$ in the definitions refers to the genus of the surface $S_{i}$.

Moreover, the fb-walk incident once or twice on one root-edge might also be incident on the other root-edge. Thereby arises the need for refinement of these partials into 


\begin{tabular}{|c|c|}
\hline \hline Partial & Counts these imbeddings in $S_{i}$ \\
\hline$d d_{i}(G, e, f)$ & $e$ and $f$ both occur on two fb-walks \\
$d s_{i}(G, e, f)$ & $e$ occurs on two fb-walks and $f$ on one fb-walk \\
$s d_{i}(G, e, f)$ & $e$ occurs on one fb-walk and $f$ on two fb-walks \\
$s s_{i}(G, e, f)$ & $e$ occurs on one fb-walk and $f$ on one fb-walk \\
\hline
\end{tabular}

Table 1: Double-root partials of $(G, e, f)$.

sub-partials. We will later see that this abstraction may necessitate an additional level of refinement to facilitate the calculation of genus distributions of double-rooted open chains. For this reason we term the sub-partials at the first level of abstraction as the first-order sub-partials. We now proceed to define these sub-partials:

\section{First-order Sub-partials of $(G, e, f)$}

The following three numbers are the sub-partials of $d d_{i}(G, e, f)$ :

$$
\begin{aligned}
d d_{i}^{0}(G, e, f)= & \begin{array}{l}
\text { the number of imbeddings of type- } d d_{i} \text { such that } \\
\text { neither fb-walk at } e \text { is incident on } f .
\end{array} \\
d d_{i}^{\prime}(G, e, f)= & \begin{array}{l}
\text { the number of imbeddings of type- } d d_{i} \text { such that } \\
\text { exactly one fb-walk at } e \text { is incident on } f .
\end{array} \\
d d_{i}^{\prime \prime}(G, e, f)= & \begin{array}{l}
\text { the number of imbeddings of type- } d d_{i} \text { such that } \\
\text { both fb-walks at } e \text { are incident on } f .
\end{array}
\end{aligned}
$$

We observe, by definition, that

$$
d d_{i}(G)=d d_{i}^{0}(G)+d d_{i}^{\prime}(G)+d d_{i}^{\prime \prime}(G)
$$

Similarly, the sub-partials of $d s_{i}(G, e, f)$ and $s d_{i}(G, e, f)$ are as follows:

$$
\left.\begin{array}{rl}
d s_{i}^{0}(G, e, f)= & \begin{array}{l}
\text { the number of imbeddings of type- } d s_{i} \text { such that } \\
\text { neither fb-walk at } e \text { is incident on } f .
\end{array} \\
d s_{i}^{\prime}(G, e, f)= & \begin{array}{l}
\text { the number of imbeddings of type- } d s_{i} \text { such that } \\
\text { exactly one fb-walk at } e \text { is incident on } f .
\end{array} \\
s d_{i}^{0}(G, e, f)= & \text { the number of imbeddings of type- } s d_{i} \text { such that } \\
& \text { the fb-walk at } e \text { is not incident on } f .
\end{array}\right)=\begin{aligned}
& \text { the number of imbeddings of type- } s d_{i} \text { such that } \\
& s d_{i}^{\prime}(G, e, f)=
\end{aligned}
$$

Thus,

$$
d s_{i}(G)=d s_{i}^{0}(G)+d s_{i}^{\prime}(G) \quad \text { and } \quad s d_{i}(G)=s d_{i}^{0}(G)+s d_{i}^{\prime}(G)
$$

Finally, the partial $s s_{i}(G, e, f)$ has these sub-partials:

$$
s s_{i}^{0}(G, e, f)=\text { the number of imbeddings of type-s } s_{i} \text { such that }
$$




$$
\begin{aligned}
s s_{i}^{1}(G, e, f)= & \begin{array}{l}
\text { the fb-walk at } e \text { is not incident on } f . \\
\text { the number of imbeddings of type- } s s_{i} \text { such that } \\
\text { removing the two occurrences of the edge } e \text { from } \\
\text { the fb-walk breaks it into two strands, exactly } \\
\text { one of which contains both occurrences of } f .
\end{array} \\
s s_{i}^{2}(G, e, f)= & \begin{array}{l}
\text { the number of imbeddings of type- } s s_{i} \text { such that } \\
\text { removing the two occurrences of the edge } e \text { from } \\
\text { the fb-walk breaks it into two strands, each } \\
\text { containing an occurrence of } f .
\end{array}
\end{aligned}
$$

Clearly,

$$
s s_{i}(G)=s s_{i}^{0}(G)+s s_{i}^{1}(G)+s s_{i}^{2}(G)
$$

The set of partials/sub-partials as defined above constitutes a partitioned genus distribution. It follows from the definition that

$$
g_{i}(G)=d d_{i}(G)+d s_{i}(G)+s d_{i}(G)+s s_{i}(G)
$$

\section{Single-root partials of $(G, e)$}

Similarly, the imbeddings of single-rooted graphs can be differentiated into two distinct types depending on whether the two occurrences of the root-edge are in the same or in different fb-walks of an imbedding. Thus, the number $g_{i}(G, e)$ is the sum of the following single-root partials:

$$
\begin{aligned}
& s_{i}(G, e)=\quad \begin{array}{l}
\text { The number of imbeddings of } G \text { such that } \\
e \text { occurs twice on the same fb-walk. }
\end{array} \\
& d_{i}(G, e)=\begin{array}{l}
\text { The number of imbeddings of } G \text { such that } \\
e \text { occurs on two different fb-walks. }
\end{array}
\end{aligned}
$$

\section{Modeling edge-amalgamation}

Let $p$ and $q$ be any of the partials such as those discussed above. Then a production expresses how an imbedding of the single-rooted graph $(G, e)$ of type $p$ on surface $S_{i}$ and an imbedding of the double-rooted graph $(H, g, f)$ of type $q$ on surface $S_{j}$ amalgamate on root-edges $e$ and $g$ to give certain types of imbeddings of the single-rooted graph $(W, f)$. This is represented as

$$
p_{i}(G) * q_{j}(H) \longrightarrow c_{1} u_{k_{1}}(W)+c_{2} v_{k_{2}}(W)+c_{3} w_{k_{3}}(W)+c_{4} z_{k_{4}}(W)
$$

where $c_{1}, c_{2}, c_{3}, c_{4}$ are integer constants and $k_{1}, k_{2}, k_{3}, k_{4}$ are integer-valued functions of $i$ and $j$. Such a production can be read as follows:

An imbedding of the graph $(G, e)$ of type $p$ on surface $S_{i}$ and an imbedding of the graph $(H, g, f)$ of type $q$ on surface $S_{j}$ amalgamate on edges $e$ and $g$ to give $c_{1}, c_{2}, c_{3}$ and $c_{4}$ imbeddings of the graph $(W, f)$ having types $u, v, w$ and $z$, respectively, on surfaces $S_{k_{1}}, S_{k_{2}}, S_{k_{3}}$ and $S_{k_{4}}$. 
Remark 3.1. Clearly, since we have 2 single-root partials for $G$ and 10 first-order doubleroot sub-partials for $H$, if we set out to derive all possible productions with these, we would need to write out 20 productions. While these are not so many in number, their derivations are fairly routine, and so we will derive only those productions that are necessary for developing our examples.

Theorem 3.2. Let $(G, e)$ be a single-edge-rooted graph and $(H, g, f)$ a double-edgerooted graph, where each of the root-edges has two 2-valent endpoints. Then the following two productions, which cover all possible cases of edge-amalgamation where the imbedding of $H$ is of type $d d^{\prime \prime}$, hold true.

$$
\begin{aligned}
& d_{i}(G) * d d_{j}^{\prime \prime}(H) \longrightarrow 2 d_{i+j}(W)+2 s_{i+j+1}(W) \\
& s_{i}(G) * d d_{j}^{\prime \prime}(H) \longrightarrow 4 d_{i+j}(W)
\end{aligned}
$$

Proof. When an imbedding of $(G, e)$ is amalgamated with an imbedding of $(H, g, f)$, the fb-walks on edges $e$ and $g$ are broken into strands that recombine into new fb-walks in the resulting imbedding of $W$, i.e., the imbedding whose rotations at all vertices are consistent with those of the imbeddings of $G$ and $H$. On the amalgamated edge there are two possibilities for the rotations at each of its two endpoints. Figure 1 demonstrates the changes in the fb-walks resulting from recombining the strands. In all four cases there is a decrease of 2 vertices and 1 edge after the amalgamation.
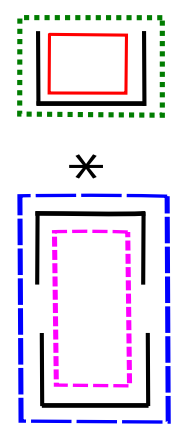
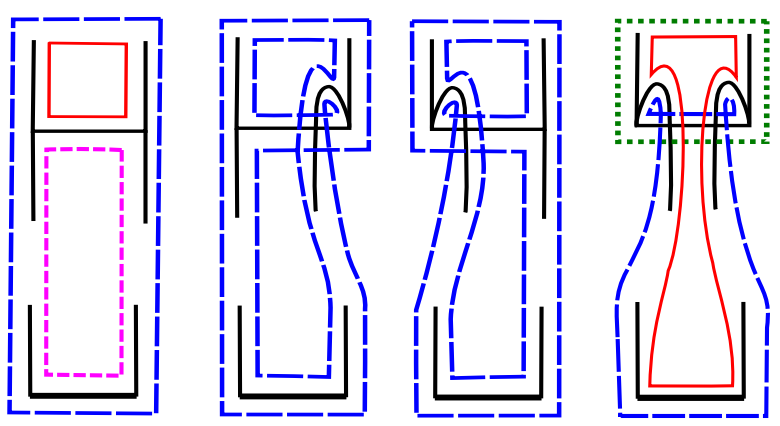

Figure 1: $d_{i}(G) * d d_{j}^{\prime \prime}(H) \longrightarrow 2 d_{i+j}(W)+2 s_{i+j+1}(W)$

The first and the last imbedding of $W$ show a decrease of 1 face, as only one fb-walk at edge $e$ combines with only one fb-walk at edge $g$. These are $d$-type imbeddings of $W$. By using the Euler polyhedral equation, we can see that the genus of the resulting imbedding of $W$ is the sum of the genera of the imbeddings of $G$ and $H$.

The second and the third imbedding of $W$ show a decrease of 3 faces as the $2 \mathrm{fb}$-walks at $e$ and the 2 at $g$ are merged into a single fb-walk. Both of these imbeddings are $s$-type imbeddings of $W$. By the Euler polyhedral equation, we can see that the genus of the resulting imbedding of $W$ is the sum of the genera of the imbeddings of $G$ and $H$ with an additional increment of one.

Production (3.2) similarly follows from the Euler polyhedral equation and yields imbeddings of type $d$ in all four cases for imbeddings of $W$ as evident from Figure 2. 

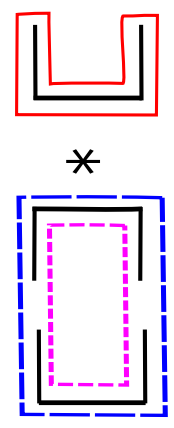
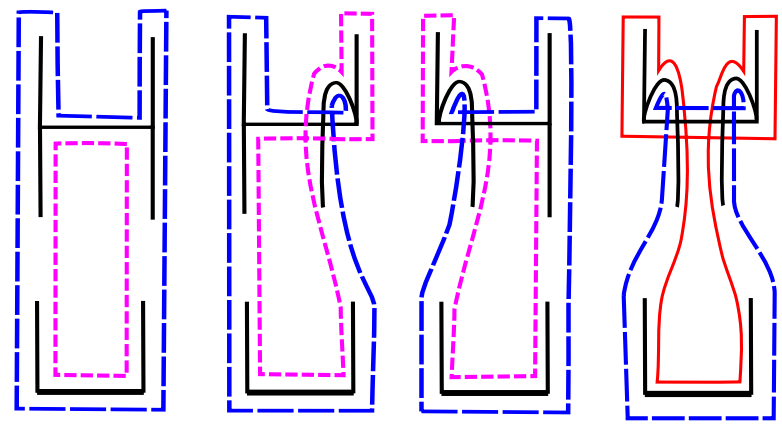

Figure 2: $s_{i}(G) * d d_{j}^{\prime \prime}(H) \longrightarrow 4 d_{i+j}(W)$

Theorem 3.3. Let $(G, e)$ be a single-edge-rooted graph and $(H, g, f)$ a double-edgerooted graph, where each of the root-edges has two 2-valent endpoints. Then the following productions, which cover all possible cases of edge-amalgamation where the imbedding of $H$ is of type $s s^{0}$ or $s s^{1}$, hold true.

$$
\begin{aligned}
& d_{i}(G) * s s_{j}^{0}(H) \longrightarrow 4 s_{i+j}(W) \\
& s_{i}(G) * s s_{j}^{0}(H) \longrightarrow 4 s_{i+j}(W) \\
& d_{i}(G) * s s_{j}^{1}(H) \longrightarrow 4 s_{i+j}(W) \\
& s_{i}(G) * s s_{j}^{1}(H) \longrightarrow 4 s_{i+j}(W)
\end{aligned}
$$

Proof. For Productions (3.3) and (3.4), the fb-walk at edge $f$ remains unaffected by the amalgamation. Thus, all four imbeddings of $W$ induced by the amalgamation of an imbedding of $G$ with an imbedding of $H$ are $s$-type imbeddings. An examination of the recombinant strands tells us that the amalgamation merges two faces incident at the root-edges. This is shown for Production (3.3) in Figure 3. Production (3.4) also has a similar illustration which we omit.
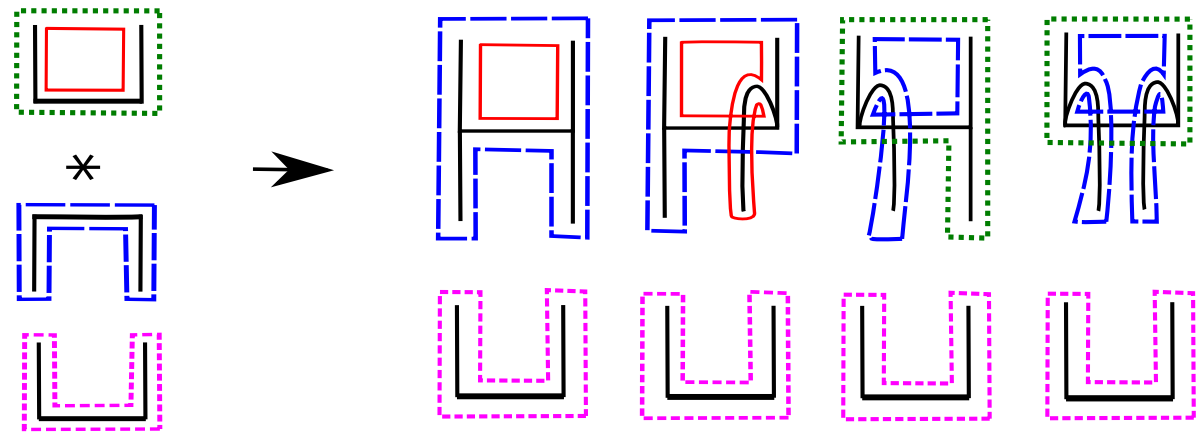

Figure 3: $d_{i}(G) * s s_{j}^{0}(H) \longrightarrow 4 s_{i+j}(W)$

The same is also true for the Productions (3.5) and (3.6). We leave the proof of Production (3.5) to the reader and demonstrate it for Production (3.6) in Figure 4. 

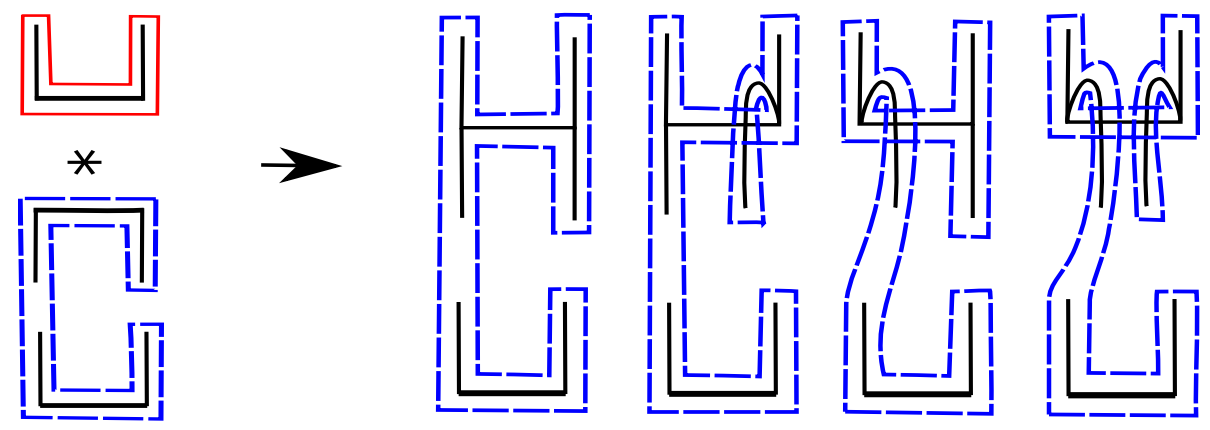

Figure 4: $s_{i}(G) * s s_{j}^{1}(H) \longrightarrow 4 s_{i+j}(W)$

Theorem 3.4. Let $(G, e)$ be a single-edge-rooted graph and $(H, g, f)$ a double-edgerooted graph, where all roots have two 2-valent endpoints. Then the following productions hold true:

$$
\begin{aligned}
& d_{i}(G) * d d_{j}^{0}(H) \longrightarrow 2 d_{i+j}(W)+2 d_{i+j+1}(W) \\
& s_{i}(G) * d d_{j}^{0}(H) \longrightarrow 4 d_{i+j}(W) \\
& d_{i}(G) * d d_{j}^{\prime}(H) \longrightarrow 2 d_{i+j}(W)+2 d_{i+j+1}(W) \\
& s_{i}(G) * d d_{j}^{\prime}(H) \longrightarrow 4 d_{i+j}(W) \\
& d_{i}(G) * d s_{j}^{0}(H) \longrightarrow 2 s_{i+j}(W)+2 s_{i+j+1}(W) \\
& s_{i}(G) * d s_{j}^{0}(H) \longrightarrow 4 s_{i+j}(W) \\
& d_{i}(G) * d s_{j}^{\prime}(H) \longrightarrow 2 s_{i+j}(W)+2 s_{i+j+1}(W) \\
& s_{i}(G) * d s_{j}^{\prime}(H) \longrightarrow 4 s_{i+j}(W) \\
& d_{i}(G) * s d_{j}^{0}(H) \longrightarrow 4 d_{i+j}(W) \\
& s_{i}(G) * s d_{j}^{0}(H) \longrightarrow 4 d_{i+j}(W) \\
& d_{i}(G) * s d_{j}^{\prime}(H) \longrightarrow 4 d_{i+j}(W) \\
& s_{i}(G) * s d_{j}^{\prime}(H) \longrightarrow 4 d_{i+j}(W) \\
& d_{i}(G) * s s_{j}^{2}(H) \longrightarrow 2 d_{i+j}(W)+2 s_{i+j}(W) \\
& s_{i}(G) * s s_{j}^{2}(H) \longrightarrow 4 s_{i+j}(W)
\end{aligned}
$$

Proof. We omit the proof for the sake of brevity.

To illustrate our technique, we present the derivation of the genus distribution of the historically significant family of closed-end ladders [5].

\section{Application: closed-end ladder}

Let $L_{0}$ be the closed-end ladder with end-rungs but no middle-rung. It is equivalent under barycentric sub-division to the four cycle $C_{4}$, with the two non-adjacent edges serving as the root-edges. Let $L_{n}$ be the closed-end ladder with $n$ middle rungs; one end-rung is trisected, and the middle third serves as a single root-edge. Thus, $L_{n}=L_{n-1} * L_{0}$ (for $n \geq 1$ ). See Figure 5 . 


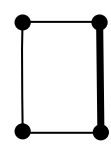

$\mathrm{L}_{0}$

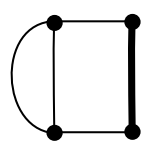

$\mathrm{L}_{1}$

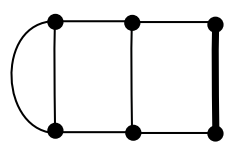

$\mathrm{L}_{2}$

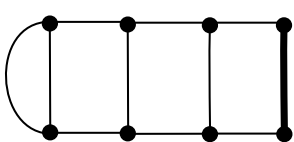

$\mathrm{L}_{3}$

Figure 5: Closed-end ladders.

Remark 4.1. For $L_{1}=L_{0} * L_{0}$, it is understood here that the first amalgamand is singlerooted whereas the second is double-rooted.

Applying the face-tracing algorithm [14] on $L_{0}$ reveals that $d d_{0}^{\prime \prime}$ is the only non-zero partial of $L_{0}$. Theorem 3.2 lists the productions necessary for edge-amalgamation when the second amalgamand is a $d d^{\prime \prime}$-type imbedding, and it has the following implications:

Theorem 4.2. Let $\left(L_{n-1}, f\right)=\left(L_{n-1}, e\right) *\left(L_{0}, g, f\right)$, where each of the root-edges e, $g, f$ has two 2-valent endpoints. Then,

$$
\begin{aligned}
& d_{k}\left(L_{n}\right)=\sum_{i=0}^{k}\left(2 d_{i}\left(L_{n-1}\right)+4 s_{i}\left(L_{n-1}\right)\right) \times d d_{k-i}^{\prime \prime}\left(L_{0}\right) \\
& s_{k}\left(L_{n}\right)=\sum_{i=0}^{k-1} 2 d_{i}\left(L_{n-1}\right) \times d d_{k-1-i}^{\prime \prime}\left(L_{0}\right)
\end{aligned}
$$

Proof. Production (3.1) indicates that amalgamating a $d$-type imbedding of the singlerooted graph $L_{n-1}$ on $S_{i}$ with a $d d^{\prime \prime}$-type imbedding of $L_{0}$ on surface $S_{j}$ induces four imbeddings of the single-rooted graph $L_{n}$, two on the surface $S_{i+j}$ and two on the surface $S_{i+j+1}$. This explains the terms $\sum_{i=0}^{k} 2 d_{i}\left(L_{n-1}\right) \times d d_{k-i}^{\prime \prime}\left(L_{0}\right)$ of Equation (4.1) and accounts for the Equation (4.2). The terms $\sum_{i=0}^{k} 4 s_{i}\left(L_{n-1}\right) \times d d_{k-i}^{\prime \prime}\left(L_{0}\right)$ of Equation (4.1) follow from the Production (3.2).

Since $d d_{i}^{\prime \prime}\left(L_{0}\right)=1$ for $i=0$ and 0 otherwise, we obtain the recurrences:

$$
\begin{aligned}
& d_{k}\left(L_{n}\right)=\left(2 d_{k}\left(L_{n-1}\right)+4 s_{k}\left(L_{n-1}\right)\right) \times d d_{0}^{\prime \prime}\left(L_{1}\right)=2 d_{k}\left(L_{n-1}\right)+4 s_{k}\left(L_{n-1}\right) \\
& s_{k}\left(L_{n}\right)=2 d_{k-1}\left(L_{n-1}\right) \times d d_{0}^{\prime \prime}\left(L_{1}\right)=2 d_{k-1}\left(L_{n-1}\right)
\end{aligned}
$$

which are analogous to the forms of recurrences obtained for cobble-stone paths in [5], and which can be solved identically to produce this formula, which was also first computed by [5].

$$
g_{i}\left(L_{n}\right)=\left\{\begin{array}{l}
2^{n-1+i}\left(\begin{array}{c}
n+1-i \\
i
\end{array}\right) \frac{2 n+2-3 i}{n+1-i} \text { for } i \leq \frac{n+1}{2} \\
0 \text { otherwise }
\end{array}\right.
$$

\section{Application: open chains of copies of $\ddot{L}_{2}$}

Let $\ddot{L}_{2}$ be the graph obtained from the ladder $L_{2}$ by trisecting the two side-rungs and designating the middle third of these trisected edges as root-edges. Let $G_{0}$ be a singlerooted graph homeomorphic to $\ddot{L}_{2}$, with the middle third of the only trisected side-rung serving as a root-edge. We can form an open chain, $G_{n}$, of copies of $\ddot{L}_{2}$ by taking $G_{n}=$ $G_{n-1} * \ddot{L}_{2}($ for $n \geq 1$ ) as shown in Figure 6. 


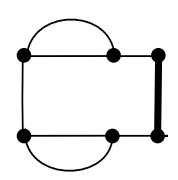

$\mathrm{G}_{0}$

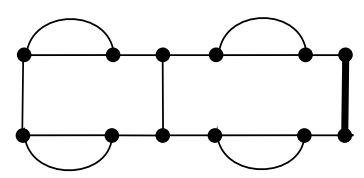

$\mathrm{G}_{1}$

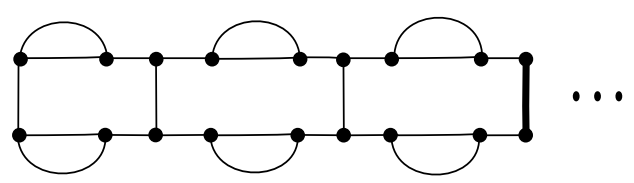

$\mathrm{G}_{2}$

Figure 6: Open chains of copies of $\ddot{L}_{2}$.

Face-tracing of $\ddot{L}_{2}$ demonstrates that its only non-zero-valued double-root first-order sub-partials are $d d_{0}^{\prime \prime}\left(\ddot{L}_{2}\right), s s_{1}^{0}\left(\ddot{L}_{2}\right)$ and $s s_{1}^{1}\left(\ddot{L}_{2}\right)$. Thus, the only productions we need for calculating the genus distribution of an open chain of copies of $\ddot{L}_{2}$ are those listed in Theorems 3.2 and 3.3. These productions make contributions to $d_{k}\left(G_{n}\right)$ or $s_{k}\left(G_{n}\right)$ as captured in the following equations:

$$
\begin{aligned}
d_{k}\left(G_{n}\right)= & \sum_{i=0}^{k}\left[2 d_{i}\left(G_{n-1}\right) * d d_{k-i}^{\prime \prime}\left(\ddot{L}_{2}\right)+4 s_{i}\left(G_{n-1}\right) * d d_{k-i}^{\prime \prime}\left(\ddot{L}_{2}\right)\right] \\
s_{k}\left(G_{n}\right)=\sum_{i=0}^{k}[ & {\left[4 d_{i}\left(G_{n-1}\right) * s s_{k-i}^{0}\left(\ddot{L}_{2}\right)+4 s_{i}\left(G_{n-1}\right) * s s_{k-i}^{0}\left(\ddot{L}_{2}\right)\right.} \\
& \left.+4 d_{i}\left(G_{n-1}\right) * s s_{k-i}^{1}\left(\ddot{L}_{2}\right)+4 s_{i}\left(G_{n-1}\right) * s s_{k-i}^{1}\left(\ddot{L}_{2}\right)\right]+ \\
& \sum_{i=0}^{k-1}\left[2 d_{i}\left(G_{n-1}\right) * d d_{k-1-i}^{\prime \prime}\left(\ddot{L}_{2}\right)\right] \\
= & \sum_{i=0}^{k}\left[4 g_{i}\left(G_{n-1}\right) * s s_{k-i}^{0}\left(\ddot{L}_{2}\right)+4 g_{i}\left(G_{n-1}\right) * s s_{k-i}^{1}\left(\ddot{L}_{2}\right)\right]+ \\
& \sum_{i=0}^{k-1}\left[2 d_{i}\left(G_{n-1}\right) * d d_{k-1-i}^{\prime \prime}\left(\ddot{L}_{2}\right)\right]
\end{aligned}
$$

\section{Genus distribution of $G_{n}$}

Since $d d_{0}^{\prime \prime}\left(\ddot{L}_{2}\right)=4, s s_{1}^{0}\left(\ddot{L}_{2}\right)=4, s s_{1}^{1}\left(\ddot{L}_{2}\right)=8$, it follows that

$$
\begin{aligned}
d_{k}\left(G_{n}\right) & =2 d_{k}\left(G_{n-1}\right) * d d_{0}^{\prime \prime}\left(\ddot{L}_{2}\right)+4 s_{k}\left(G_{n-1}\right) * d d_{0}^{\prime \prime}\left(\ddot{L}_{2}\right) \\
s_{k}\left(G_{n}\right) & =4 g_{k-1}\left(G_{n-1}\right) * s s_{1}^{0}\left(\ddot{L}_{2}\right)+4 g_{k-1}\left(G_{n-1}\right) * s s_{1}^{1}\left(\ddot{L}_{2}\right) \\
& +2 d_{k-1}\left(G_{n-1}\right) * d d_{0}^{\prime \prime}\left(\ddot{L}_{2}\right) \\
\Longrightarrow & \\
d_{k}\left(G_{n}\right) & =8 g_{k}\left(G_{n-1}\right)+8 s_{k}\left(G_{n-1}\right) \\
s_{k}\left(G_{n}\right) & =48 g_{k-1}\left(G_{n-1}\right)+8 d_{k-1}\left(G_{n-1}\right)
\end{aligned}
$$

As $\ddot{L}_{2} \cong G_{0}$, the partitioned genus distribution of $\ddot{L}_{2}$ implies that $d_{0}\left(G_{0}\right)=4$ and $s_{1}\left(G_{0}\right)=12$. Therefore, we can iteratively plug values into Equations (5.1) and (5.2), and thereby calculate the genus distributions given in Tables $2-4$. 


\begin{tabular}{|c|c|c|c|c|}
\hline \hline$k$ & $k=0$ & $k=1$ & $k=2$ & $k \geq 3$ \\
\hline$d_{k}\left(G_{1}\right)$ & 32 & 192 & 0 & 0 \\
\hline$s_{k}\left(G_{1}\right)$ & 0 & 224 & 576 & 0 \\
\hline$g_{k}\left(G_{1}\right)$ & 32 & 416 & 576 & 0 \\
\hline \hline
\end{tabular}

Table 2: Genus distribution of $G_{1}$.

\begin{tabular}{|c|c|c|c|c|c|}
\hline \hline$k$ & $k=0$ & $k=1$ & $k=2$ & $k=3$ & $k \geq 4$ \\
\hline$d_{k}\left(G_{2}\right)$ & 256 & 5120 & 9216 & 0 & 0 \\
\hline$s_{k}\left(G_{2}\right)$ & 0 & 1792 & 21504 & 27648 & 0 \\
\hline$g_{k}\left(G_{2}\right)$ & 256 & 6912 & 30720 & 27648 & 0 \\
\hline
\end{tabular}

Table 3: Genus distribution of $G_{2}$.

\begin{tabular}{|c|c|c|c|c|c|c|}
\hline \hline$k$ & $k=0$ & $k=1$ & $k=2$ & $k=3$ & $k=4$ & $k \geq 5$ \\
\hline$d_{k}\left(G_{3}\right)$ & 2048 & 69632 & 417792 & 442368 & 0 & 0 \\
\hline$s_{k}\left(G_{3}\right)$ & 0 & 14336 & 372736 & 1548288 & 1327104 & 0 \\
\hline$g_{k}\left(G_{3}\right)$ & 2048 & 83968 & 790528 & 1990656 & 1327104 & 0 \\
\hline \hline
\end{tabular}

Table 4: Genus distribution of $G_{3}$.

Remark 5.1. From Tables $2-4$, the genus distributions for open chains of $\ddot{L}_{2}$ appear to support the unimodality conjecture that all graphs have unimodal genus distributions. The amalgamation approach is likely to be useful in such contexts either by producing counterexamples to the conjecture or by providing recurrences like Equations (5.1) and (5.2) which may be instrumental in proving unimodality for certain families of graphs.

\section{Non-homeomorphic graphs with identical genus distributions}

The earliest published example for non-homeomorphic graphs with identical genus distributions is given in [12]. [20] provides a more general method for generating such pairs. We now discuss a simpler method for constructing such examples.

There are two ways of edge-amalgamating the graphs $(G, e)$ and $(H, f)$, depending on how the endpoints of the root-edges $e$ and $f$ are paired. We observe that all the productions for edge-amalgamation in Theorems 3.2 - 3.4 are independent of how the endpoints of the respective root-edges are paired, that is, they are true for both possible pairings. Thus, for both ways of pasting, we get the same genus distribution.

One can exploit this fact to construct pairs of non-homeomorphic graphs having the same genus distribution. For instance, Figure 7 shows two non-homeomorphic graphs resulting from the two ways of edge-amalgamating the same graphs. They have the same genus distributions. To prove that they are non-isomorphic, consider the set of distances between the two double adjacencies. Since these two graphs are 3-regular, they are also non-homeomorphic. 

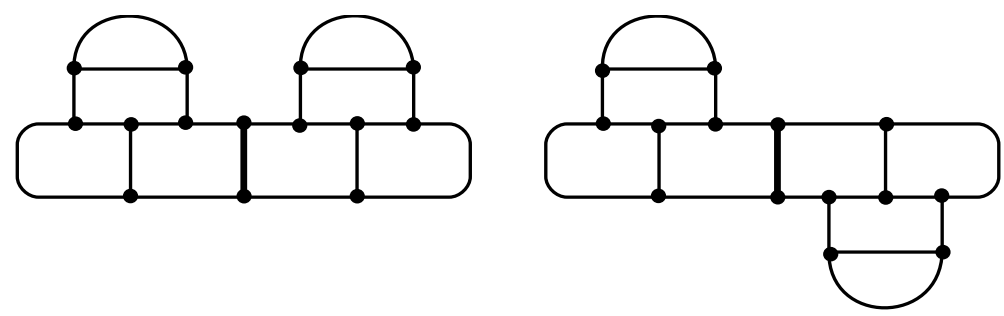

Figure 7: Non-homeomorphic graphs with the same genus distribution: $32+928 g+$ $6720 g^{2}+7680 g^{3}+1024 g^{4}$.

\section{Second-order sub-partials}

The first-order sub-partials that can be further partitioned into second-order sub-partials are characterized by having an fb-walk incident on both roots, but not on all four occurrences of these roots. In particular, these are $d d^{\prime}, d d^{\prime \prime}, d s^{\prime}$ and $s d^{\prime}$. In order to describe second-order sub-partials, we imagine a "thickening" of the root-edges of the graph $(G, e, f)$, and we label the two "sides" of the thickened edge $e$ as 1 and 2, and the two sides of the thickened edge $f$ as 3 and 4, as shown in Figure 8.

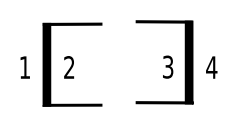

Figure 8: Modeling second-order sub-partials.

Distinguishing which of these labeled sides come together in an fb-walk is an important piece of information, which we would like to capture in our second-order sub-partial, as it is essential for double-rooted edge-amalgamation. Thus, for example, a $d d^{\prime}$-type imbedding may combine the faces 1 and 3, faces 1 and 4, faces 2 and 3, or faces 2 and 4. Accordingly, we define the second-order sub-partials for $d d^{\prime}$ as illustrated in the top half of Figure 9. We show the remaining sub-partials in the bottom half of the figure. We thus define the second-order sub-partials as follows:

$$
\begin{aligned}
& {\overline{d d^{\prime}}}_{i}(G, e, f)=\text { the number of imbeddings of type- } d d_{i}^{\prime} \text { such that } \\
& \text { the sides } 1 \text { and } 4 \text { occur in the same fb-walk. } \\
& \widetilde{d d^{\prime}}{ }_{i}(G, e, f)=\text { the number of imbeddings of type- } d d_{i}^{\prime} \text { such that } \\
& \text { the sides } 2 \text { and } 3 \text { occur in the same fb-walk. } \\
& {\overrightarrow{d d^{\prime}}}_{i}(G, e, f)=\text { the number of imbeddings of type- } d d_{i}^{\prime} \text { such that } \\
& \text { the sides } 1 \text { and } 3 \text { occur in the same fb-walk. } \\
& {\overleftarrow{d d^{\prime}}}_{i}(G, e, f)=\text { the number of imbeddings of type- } d d_{i}^{\prime} \text { such that }
\end{aligned}
$$

Similarly,

$$
\begin{aligned}
{\overrightarrow{d d^{\prime \prime}}}_{i}(G, e, f)= & \text { the number of imbeddings of type- } d d_{i}^{\prime \prime} \text { such that } \\
& \text { the sides } 1 \text { and } 4 \text { occur in the same fb-walk and } \\
& \text { the sides } 2 \text { and } 3 \text { in another. }
\end{aligned}
$$




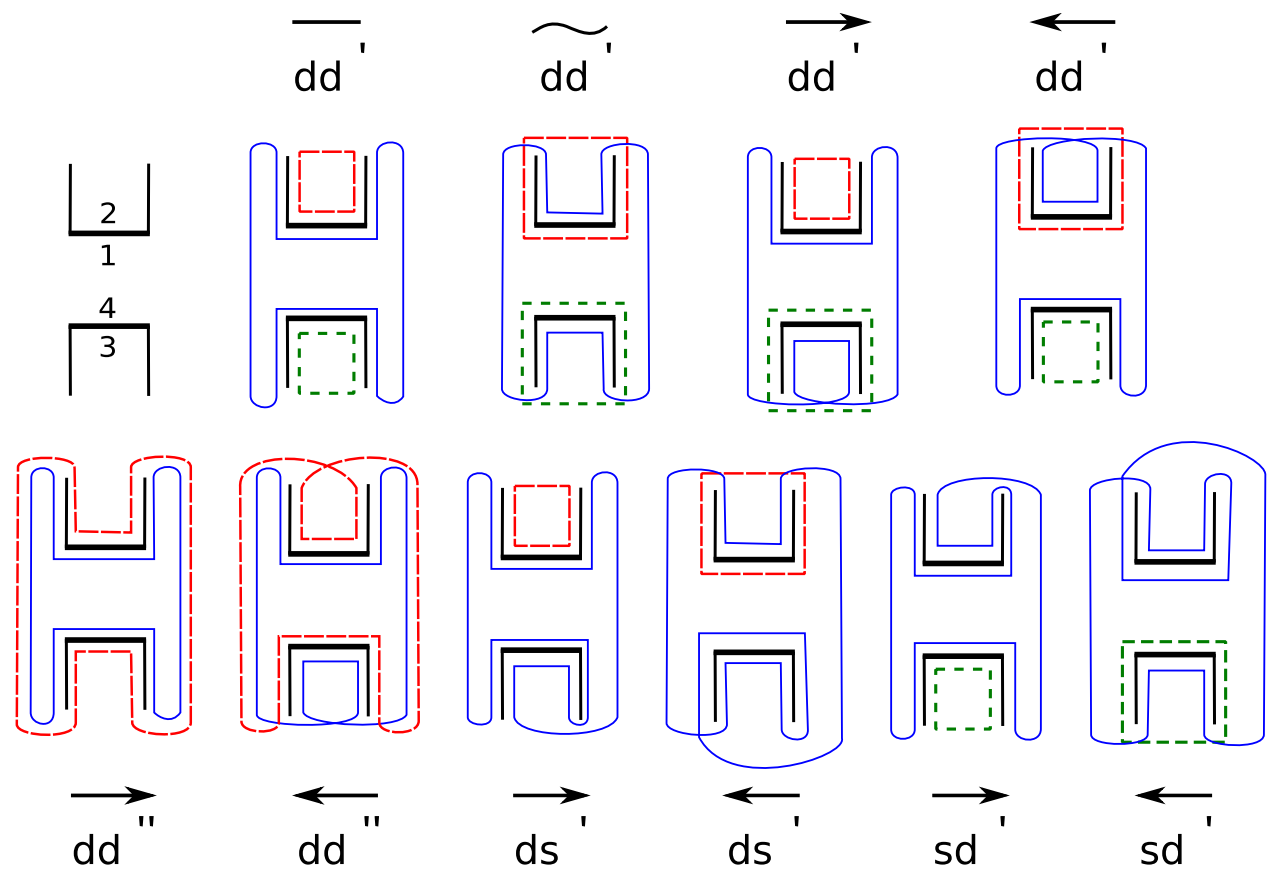

Figure 9: Models for second-order sub-partials.

$$
\begin{aligned}
{\overleftarrow{d d^{\prime \prime}}}_{i}(G, e, f)= & \text { the number of imbeddings of type- } d d_{i}^{\prime \prime} \text { such that } \\
& \text { the sides } 1 \text { and } 3 \text { occur in the same fb-walk and } \\
& \text { the sides } 2 \text { and } 4 \text { in another. }
\end{aligned}
$$

And finally,

$$
\begin{aligned}
{\overrightarrow{d s^{\prime}}}_{i}(G, e, f)= & \begin{array}{l}
\text { the number of imbeddings of type- } d s_{i}^{\prime} \text { such that } \\
\text { the sides } 1,3,4 \text { occur in the same fb-walk. }
\end{array} \\
{\overleftarrow{d s^{\prime}}}_{i}(G, e, f)= & \begin{array}{l}
\text { the number of imbeddings of type- } d s_{i}^{\prime} \text { such that } \\
\text { the sides } 2,3,4 \text { occur in the same fb-walk. }
\end{array} \\
{\overrightarrow{s d^{\prime}}}_{i}(G, e, f)= & \begin{array}{l}
\text { the number of imbeddings of type- } s d_{i}^{\prime} \text { such that } \\
\text { the sides } 1,2,4 \text { occur in the same fb-walk. }
\end{array} \\
{\overleftarrow{s d^{\prime}}}_{i}(G, e, f)= & \begin{array}{l}
\text { the number of imbeddings of type- } s d_{i}^{\prime} \text { such that } \\
\text { the sides } 1,2,3 \text { occur in the same fb-walk. }
\end{array}
\end{aligned}
$$

A complete list of productions for edge-amalgamation using only double-root partials can be derived in a manner akin to our method in $\S 3$. One could work out all $16 \times 16=256$ productions by using the first-order sub-partials and substituting the use of $d d^{\prime}, d d^{\prime \prime}, d s^{\prime}$ and $s d^{\prime}$ by their respective second-order sub-partials defined in this section. For the sake of brevity, we list in Table 5 only the productions needed for the first of our target applications in $\S 8$ and outline their proofs on our website [33]. We abbreviate the partials through omission of the graphs $G, H$ and $W$. 


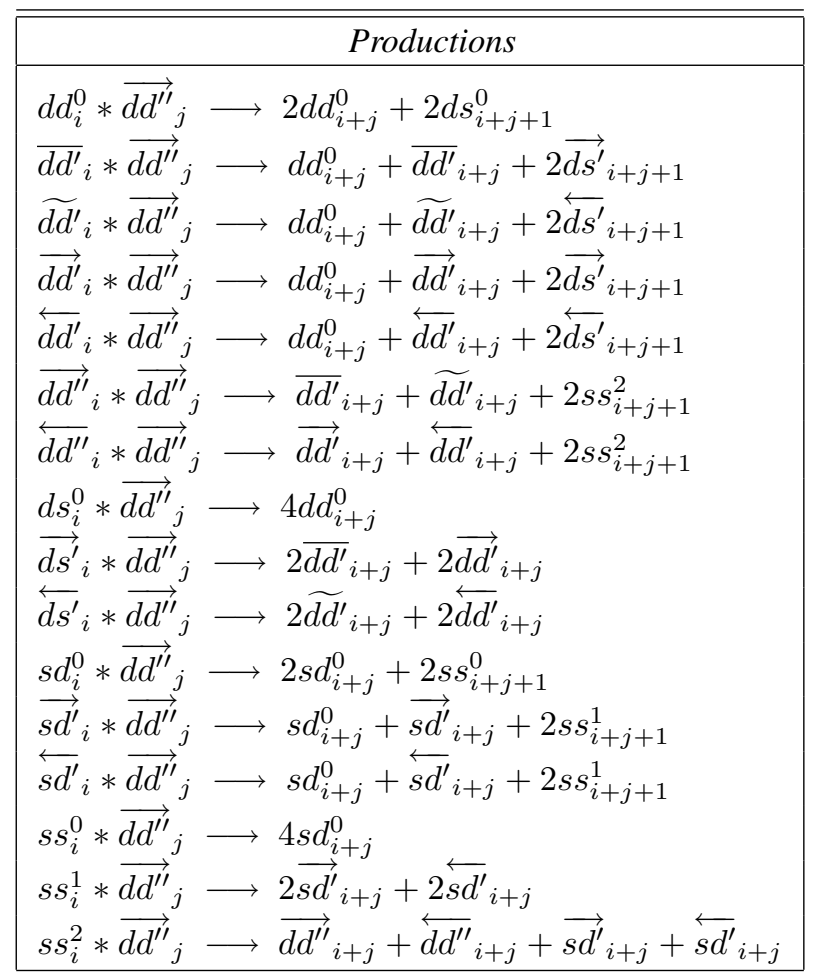

Table 5: A subset of the productions for the edge-amalgamation $(G, e, d) *(H, g, f)$.

In general, when amalgamating copies of a base graph, some of the partials of the base graph may be zero-valued. Accordingly, we can eliminate a lot of unnecessary work and use this good fortune to derive a smaller subset of productions relevant to our particular application. The productions in Table 5 lead to Theorem 7.1.

Theorem 7.1. Let $(W, e, f)=(G, e, d) *(H, g, f)$, where each of the root-edges $e, d, g, f$ has two 2-valent endpoints and the imbeddings of the graph $H$ are of type $\overrightarrow{d d^{\prime \prime}}$. Then,

$$
\begin{aligned}
d d_{k}^{0}(W) & =\sum_{i=0}^{k}\left(2 d d_{i}^{0}(G)+d d_{i}^{\prime}(G)+4 d s_{i}^{0}(G)\right) \times{\overrightarrow{d d^{\prime \prime}}}_{k-i}(H) \\
{\overrightarrow{d d^{\prime}}}_{k}(W) & =\sum_{i=0}^{k}\left({\overrightarrow{d d^{\prime}}}_{i}(G)+{\overrightarrow{d d^{\prime \prime}}}_{i}(G)+2{\overrightarrow{d s^{\prime}}}_{i}(G)\right) \times{\overrightarrow{d d^{\prime \prime}}}_{k-i}(H) \\
{\widetilde{d d^{\prime}}}_{k}(W) & =\sum_{i=0}^{k}\left({\widetilde{d d^{\prime}}}_{i}(G)+{\overrightarrow{d d^{\prime \prime}}}_{i}(G)+2{\overleftrightarrow{d s^{\prime}}}_{i}(G)\right) \times{\overrightarrow{d d^{\prime \prime}}}_{k-i}(H) \\
{\overrightarrow{d d^{\prime}}}_{k}(W) & =\sum_{i=0}^{k}\left({\overrightarrow{d d^{\prime}}}_{i}(G)+{\overleftrightarrow{d d^{\prime \prime}}}_{i}(G)+2{\overrightarrow{d s^{\prime}}}_{i}(G)\right) \times{\overrightarrow{d d^{\prime \prime}}}_{k-i}(H) \\
{\overleftrightarrow{d d^{\prime}}}_{k}(W) & =\sum_{i=0}^{k}\left({\overleftrightarrow{d d^{\prime}}}_{i}(G)+{\overleftrightarrow{d d^{\prime \prime}}}_{i}(G)+2{\overleftrightarrow{d s^{\prime}}}_{i}(G)\right) \times{\overrightarrow{d d^{\prime \prime}}}_{k-i}(H) \\
{\overrightarrow{d d^{\prime \prime}}}_{k}(W) & =\sum_{i=0}^{k} s s_{i}^{2}(G) \times{\overrightarrow{d d^{\prime \prime}}}_{k-i}(H) \\
{\overleftrightarrow{d d^{\prime \prime}}}_{k}(W) & =\sum_{i=0}^{k} s s_{i}^{2}(G) \times{\overrightarrow{d d^{\prime \prime}}}_{k-i}(H)
\end{aligned}
$$




$$
\begin{aligned}
d s_{k}^{0}(W) & =\sum_{i=0}^{k-1} 2 d d_{i}^{0}(G) \times{\overrightarrow{d d^{\prime \prime}}}_{k-1-i}(H) \\
{\overrightarrow{d s^{\prime}}}_{k}(W) & =\sum_{i=0}^{k-1} 2\left({\overrightarrow{d d^{\prime}}}_{i}(G)+{\overrightarrow{d d^{\prime}}}_{i}(G)\right) \times{\overrightarrow{d d^{\prime \prime}}}_{k-1-i}(H) \\
{\overleftarrow{d s^{\prime}}}_{k}(W) & =\sum_{i=0}^{k-1} 2\left({\widetilde{d d^{\prime}}}_{i}(G)+{\overleftrightarrow{d d^{\prime}}}_{i}(G)\right) \times{\overrightarrow{d d^{\prime \prime}}}_{k-1-i}(H) \\
s d_{k}^{0}(W) & =\sum_{i=0}^{k}\left(2 s d_{i}^{0}(G)+s{d_{i}^{\prime}}_{i}(G)+4 s s_{i}^{0}(G)\right) \times{\overrightarrow{d d^{\prime \prime}}}_{k-i}(H) \\
\overrightarrow{s d}^{\prime} & (W)=\sum_{i=0}^{k}\left({\overrightarrow{s d^{\prime}}}_{i}(G)+2 s s_{i}^{1}(G)+s s_{i}^{2}(G)\right) \times{\overrightarrow{d d^{\prime \prime}}}_{k-i}(H) \\
{\overleftarrow{s d^{\prime}}}_{k}(W) & =\sum_{i=0}^{k}\left({\overleftarrow{s d^{\prime}}}_{i}(G)+2 s s_{i}^{1}(G)+s s_{i}^{2}(G)\right) \times{\overrightarrow{d d^{\prime \prime}}}_{k-i}(H) \\
s s_{k}^{0}(W) & =\sum_{i=0}^{k-1} 2 s d_{i}^{0}(G) \times{\overrightarrow{d d^{\prime \prime}}}_{k-1-i}(H) \\
s s_{k}^{1}(W) & =\sum_{i=0}^{k-1} 2 s d_{i}^{\prime}(G) \times{\overrightarrow{d d^{\prime \prime}}}_{k-1-i}(H) \\
s s_{k}^{2}(W) & =\sum_{i=0}^{k-1} 2 d d_{i}^{\prime \prime}(G) \times{\overrightarrow{d d^{\prime \prime}}}_{k-1-i}(H)
\end{aligned}
$$

Proof. Consider the production:

$$
d d_{i}^{0}(G) *{\overrightarrow{d d^{\prime \prime}}}_{j}(H) \longrightarrow 2 d d_{i+j}^{0}(W)+2 d s_{i+j+1}^{0}(W)
$$

It indicates that each $d d^{0}$-type imbedding of $G$ on $S_{i}$ when amalgamated with a $\overrightarrow{d d^{\prime \prime}}$-type imbedding of $H$ on surface $S_{j}$, induces two imbeddings of $W$ having type $d d^{0}$ on surface $S_{i+j}$ and two of type $d s^{0}$ on surface $S_{i+j+1}$.

These contributions account for the term $\sum_{i=0}^{k} 2 d d_{i}^{0} \times d d_{k-i}^{\prime \prime}$ in Equation (7.1) and for the Equation (7.8). Taking into account all contributions made by the productions in Table 5 , the result follows.

\section{Application: closed-end ladders}

We showed in $\S 4$ how to compute the single-root partials for the genus distribution of closed-end ladders. We can accomplish the same for double-root partials of closed-end ladders.

Remark 8.1. In [22], we use these double-root partials for calculating genus distributions of closed chains which are "cycles" of copies of a given base graph. The two closed chains corresponding to closed-end ladders are circular ladders and Möbius ladders.

By face-tracing we know that all partials for $L_{0}$ are zero-valued except for ${\overrightarrow{d d^{\prime \prime}}}_{0}\left(L_{0}\right)$, whose value is 1 . This is the vital piece of information which we utilized in selecting the 16 productions that we chose to derive for this application and that we listed in Table 5, from amongst a total of 256 productions. We can use the value of this partial and iteratively apply Theorem 7.1 to obtain the partitioned genus distribution for the closed-end ladders. The reader will observe that the values for $g_{k}\left(L_{n}\right)$ agree with the values first obtained by [5]. For the sake of completion, we include the table of partitioned genus distributions for $L_{0}$ through $L_{5}$ in [33].

The reader may also observe that the same results could have also been achieved using first-order sub-partials and may question the need for using second-order sub-partials for 
amalgamating double-rooted graphs. However, in general, with more complex applications requiring amalgamations of double-rooted graphs having higher degrees, one is likely to need the additional information captured in second-order sub-partials. One such application is calculating the genus distribution of an open chain of copies of the complete bipartite graph $K_{3,3}$.

\section{Application: open chains of copies of $K_{3,3}$}

We omit the proof and list only the results computed by using our technique. In particular, we list only the non-zero columns.
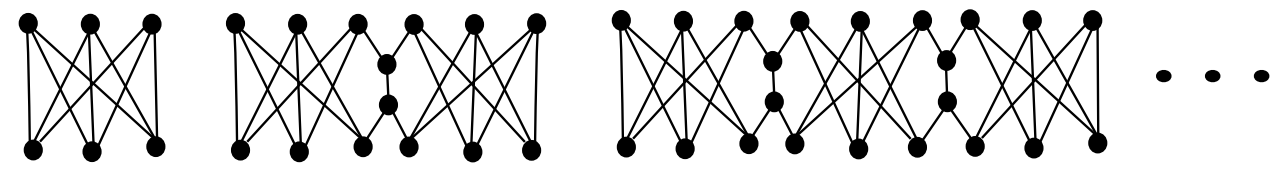

Figure 10: Open chains $G_{0}=K_{3,3}, G_{1}=G_{0} * K_{3,3}, G_{2}=G_{1} * K_{3,3}$.

\begin{tabular}{|c|c|c|c|c|c|c|c|c|c|}
\hline$G_{n}$ & \multicolumn{2}{|c|}{$G_{0}$} & \multicolumn{3}{|c|}{$G_{1}$} & \multicolumn{4}{|c|}{$G_{2}$} \\
\hline$k$ & 1 & 2 & 2 & 3 & 4 & 3 & 4 & 5 & 6 \\
\hline$d d_{k}^{0}$ & 0 & 0 & 1656 & 0 & 0 & 262976 & 436224 & 0 & 0 \\
\hline$\overline{d{d d^{\prime}}^{n}}$ & 4 & 0 & 344 & 440 & 0 & 13296 & 78064 & 31040 & 0 \\
\hline${\widetilde{d d^{\prime}}}_{k}$ & 4 & 0 & 344 & 440 & 0 & 13296 & 78064 & 31040 & 0 \\
\hline $\overrightarrow{d d^{\prime}}{ }_{k}$ & 6 & 0 & 280 & 440 & 0 & 13808 & 78064 & 31040 & 0 \\
\hline$\overleftarrow{d d^{\prime}}{ }_{k}$ & 6 & 0 & 280 & 440 & 0 & 13808 & 78064 & 31040 & 0 \\
\hline${\overrightarrow{d d^{\prime \prime}}}_{k}$ & 0 & 0 & 24 & 144 & 0 & 144 & 2160 & 5184 & 0 \\
\hline$\overbrace{d d^{\prime \prime}}$ & 6 & 0 & 24 & 144 & 0 & 144 & 2160 & 5184 & 0 \\
\hline$d s_{k}^{0}$ & 2 & 0 & 424 & 1040 & 0 & 58784 & 339488 & 171392 & 0 \\
\hline $\overrightarrow{d s^{\prime}}{ }_{k}$ & 2 & 0 & 104 & 1016 & 0 & 4384 & 68256 & 158336 & 0 \\
\hline${\overleftarrow{d s^{\prime}}}_{k}$ & 2 & 0 & 104 & 1016 & 0 & 4384 & 68256 & 158336 & 0 \\
\hline$s d_{k}^{0}$ & 2 & 0 & 424 & 1040 & 0 & 58784 & 339488 & 171392 & 0 \\
\hline $\overrightarrow{s d^{\prime}} k$ & 2 & 0 & 116 & 1088 & 0 & 4336 & 68688 & 160064 & 0 \\
\hline$\overleftarrow{s d^{\prime}}{ }_{k}$ & 2 & 0 & 92 & 944 & 0 & 4432 & 67824 & 156608 & 0 \\
\hline$s s_{k}^{0}$ & 0 & 0 & 96 & 664 & 0 & 12928 & 133312 & 280576 & 0 \\
\hline$s s_{k}^{1}$ & 0 & 12 & 32 & 704 & 2016 & 1408 & 36416 & 230336 & 214272 \\
\hline$s s_{k}^{2}$ & 2 & 12 & 8 & 168 & 288 & 32 & 1440 & 8640 & 6912 \\
\hline$g_{k}$ & 40 & 24 & 4352 & 9728 & 2304 & 466944 & 1875968 & 1630208 & 221184 \\
\hline
\end{tabular}

Table 6: Genus distributions of open chains of copies of $K_{3,3}$. 


\section{Conclusions}

The methods highlighted in this paper enable us to formulate recurrences that specify the partitioned genus distributions for an arbitrarily large graph, constructed by iteratively linking smaller graph units of known partitioned genus distributions on root-edges that have two 2-valent endpoints. These smaller graphs may have arbitrarily large degrees at vertices on which the root-edges are not incident. In this manner, one can construct open chains consisting of copies of the same graph. Similarly, one can interleave copies of many distinct graphs. We learn how to do this efficiently by using single-root partials and doubleroot first-order sub-partials. We also introduce second-order sub-partials in the interest of accomplishing the same using only double-root sub-partials. This enables us to lay the ground for self-amalgamation, which we cover in the second installment of this paper [22].

We examine as applications of our techniques open chains of copies of the closedend ladder $\ddot{L}_{2}$ and of $K_{3,3}$. In revisiting the closed-end ladders, we bring to the reader's attention how, in some cases, it may be possible to solve the recurrences and obtain closed formulas. We discuss how the results in this paper can aid one in generating examples of non-homeomorphic pairs of graphs that have identical genus distributions.

Moreover, in combination with methods from [8], we can develop the relationship of the partitioned genus distribution of edge-linked open chains to other graphs by exploring edge operations such as contracting, splitting, edge-addition and edge-deletion. The techniques developed here can also be used creatively as in [9], which presents a quadratictime algorithm for computing the genus distribution of any cubic outerplanar graph. These techniques are also likely to be helpful in finding a counterexample to the unimodality conjecture, if such a counterexample exists.

Further avenues for research include edge-amalgamation on roots with higher-valent endpoints, and analysis of the recurrences for properties such as the unimodality of genus distributions.

\section{References}

[1] L. W. Beineke, R. J. Wilson, J. L. Gross and T. W. Tucker (eds), Topics in Topological Graph Theory, Cambridge University Press, Cambridge, UK, 2009.

[2] C. P. Bonnington, M. J. Grannell, T. S. Griggs and J. Širáň, Exponential families of nonisomorphic triangulations of complete graphs, J. Combin. Theory (B) 78 (2000), 169-184.

[3] J. Chen, J. L. Gross and R. G. Rieper, Overlap matrices and total imbedding distributions, Discrete Math. 128 (1994), 73-94.

[4] Y. C. Chen, Y. P. Liu and T. Wang, The total embedding distributions of cacti and necklaces, Acta Math. Sinica - English Series 22 (2006), 1583-1590.

[5] M. L. Furst, J. L. Gross and R. Statman, Genus distribution for two classes of graphs, J. Combin. Theory (B) 46 (1989), 22-36.

[6] L. Goddyn, R. B. Richter and J. Širáň, Triangular embeddings of complete graphs from graceful labellings of paths, J. Combin. Theory (B) 97 (2007), 964-970.

[7] M. J. Grannell and T. S. Griggs, A lower bound for the number of triangular embeddings of some complete graphs and complete regular tripartite graphs, J. Combin. Theory (B) 98 (2008), 637-650.

[8] J. L. Gross, Edge-operation effect on genus distribution. Joining, deleting, contracting, splitting, preprint, 2010, 28 pages. 
[9] J. L. Gross, Genus distributions of cubic outerplanar graphs, preprint, 2010, 23 pages.

[10] J. L. Gross and M. L. Furst, Hierarchy for imbedding-distribution invariants of a graph, $J$. Graph Theory 11 (1987), 205-220.

[11] J. L. Gross, I. F. Khan and M. I. Poshni, Genus of graph amalgamations: pasting at rootvertices, Ars Combin. 94 (2010), 33-53.

[12] J. L. Gross, E. W. Klein and R. G. Rieper, On the average genus of a graph, Graphs and Combinatorics 9 (1993), 153-162.

[13] J. L. Gross, D. P. Robbins and T. W. Tucker, Genus distributions for bouquets of circles, $J$. Combin. Theory (B) 47 (1989), 292-306.

[14] J. L. Gross and T. W. Tucker, Topological Graph Theory, Wiley, New York, 1987, and Dover, Mineola, NY, 2001.

[15] V. P. Korzhik and H-J. Voss, Exponential families of non-isomorphic non-triangular orientable genus embeddings of complete graphs, J. Combin. Theory (B) 86 (2002), 86-211.

[16] J. H. Kwak and J. Lee, Genus polynomials of dipoles, Kyungpook Math. J. 33 (1993), 115-125.

[17] J. H. Kwak and J. Lee, Enumeration of graph embeddings, Discrete Math. 135 (1994), 129 151.

[18] J. H. Kwak and S. H. Shim, Total embedding distributions for bouquets of circles, Discrete Math. 248 (2002), 93-108.

[19] L. A. McGeoch, Algorithms for two graph problems: computing maximum-genus imbedding and the two-server problem, $\mathrm{PhD}$ thesis, Carnegie-Mellon University, 1987.

[20] L. A. McGeoch, Genus Distribution for circular and Möbius ladders., Technical report extracted from $\mathrm{PhD}$ thesis, Carnegie-Mellon University, 1987.

[21] B. P. Mull, Enumerating the orientable 2-cell imbeddings of complete bipartite graphs, J. Graph Theory 30 (1999), 77-90.

[22] M. I. Poshni, I. F. Khan, and J. L. Gross, Genus distributions of graphs under self-edgeamalgamations, preprint, 2010, 28 pages.

[23] R. G. Rieper, The enumeration of graph embeddings, $\mathrm{PhD}$ thesis, Western Michigan University, 1990.

[24] A. Riskin, On the enumeration of polyhedral embeddings of Cartesian products of cycles, Ars Combin. 41 (1995), 193-198.

[25] S. Stahl, Region distributions of graph embeddings and Stirling numbers, Discrete Math. 82 (1990), 57-78.

[26] S. Stahl, Permutation-partition pairs III: Embedding distributions of linear families of graphs, J. Combin. Theory (B) 52 (1991), 191-218.

[27] S. Stahl, Region distributions of some small diameter graphs, Discrete Math. 89 (1991), 281299.

[28] E. H. Tesar, Genus distribution of Ringel ladders, Discrete Math. 216 (2000) 235-252.

[29] T. I. Visentin and S. W. Wieler, On the genus distribution of $(p, q, n)$-dipoles, Electronic J. of Combin. 14 (2007), \#R12.

[30] L. X. Wan and Y. P. Liu, Orientable embedding distributions by genus for certain types of graphs, Ars Combin. 79 (2006), 97-105.

[31] L. X. Wan and Y. P. Liu, Orientable embedding genus distribution for certain types of graphs, J. Combin. Theory (B) 47 (2008), 19-32. 
[32] A. T. White, Graphs of Groups on Surfaces, North-Holland, Amsterdam, 2001.

[33] http://www.cs.columbia.edu/ gross/supp-edge-amalg-I.pdf 